\title{
Microbial Diversity of Ticks And A Novel Tyhpus Group Rickettsia Species ( $R$. bacterium Ac37b) Detected In Inner Mongolia, China
}

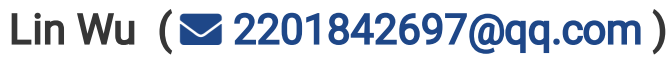

Inner Mongolia Medical University

\section{Li-li Xing}

Inner Mongolia Medical University

Zheng Gui

University of Chinese Academy of Sciences

Si Su

Inner Mongolia Medical University

Jing-feng Yu

Inner Mongolia Medical University

Dong-dong Qi

Hulunbuir Mental Health Center

Shao-yin Fu

Inner Mongolia Academy of Agricultural and Animal Husbandry Sciences

Xiao-yan Si

Inner Mongolia Center for Disease Control and Prevention

Lan $\mathrm{Mu}$

Inner Mongolia Medical University

\section{Research}

Keywords: Inner Monglia,Dermacentor nuttalli,I.persuleatus,Microbial diversity ,R.bacterium Ac37b

Posted Date: November 10th, 2021

DOI: https://doi.org/10.21203/rs.3.rs-1052401/v1

License: (c) (1) This work is licensed under a Creative Commons Attribution 4.0 International License. Read Full License 


\section{Abstract}

Background: Ticks are arthropods that can carry multiple pathogens and parasitize on livestock and mammals as well as on humans. Animal husbandry in Inner Mongolia, China, provides a suitable tick habitat. In this study, PacBio full-length 16S rDNA third-generation sequencing was used to analyze the diversity of microbial communities carried by ticks in different regions of Inner Mongolia. The aim of the study is to characterize the microbiome carried by ticks in different geographical locations and to provide theoretical support for regional prevention and control of pathogen populations in the future.

Methods: In this study, a total of 905 Dermacentor nuttalli and 36 Ixodes persuleatus were collected from the surface of sheep in four main pasture areas in Inner Mongolia. Pooled DNA samples were prepared from three samples from each region and from each tick species. In total the microbial diversity of 12 samples was analyzed by PacBio full-length 16S rRNA third-generation sequencing, and the $\alpha$ and $\beta$ diversity were determined.

Results: The main bacterial genera we found were Rickettsia (35.27\%), Ac37b (19.33\%), Arsenophonus (11.21\%), Candidatus Lariskella (10.84\%), and Acinetobacter (7.17\%). There were significant differences in the microbial composition of ticks from different regions and in different tick species. Rickettsia bellii was found in the I. persuleatus group. In addition, Anaplasma and a novel tyhpus group Rickettsia species ( $R$. bacterium Ac37b) were found in the sample group of $D$. nuttalli in the city of Ordos.

Conclusions: In this study, Rickettsia bellii was first found in I. persuleatus in Inner Mongolia, and a novel tyhpus group Rickettsia species ( $R$. bacterium Ac37b) was found in $D$. nuttalli from the city of Ordos. Our study provides a basis for the prevention and control of tick-borne diseases through the analysis of tick microbial diversity in different regions of Inner Mongolia. Furthermore, we were able to detect a new tickborne pathogen in D. nutalli.

\section{Background}

Ticks are a kind of arachnids (Arachnida: Acari: Ixodida) parasitic on animals or humans. The larvae, nymphs, and male and female adults of ticks are all bloodsucking. The hosts include terrestrial mammals, birds, reptiles, and amphibians. When ticks bite the host, they can not only cause irritation, anemia, local or systemic hypersensitivity but also become the vector for the transmission of various pathogens [1]. The pathogens transmitted by ticks are even more diverse than in mosquitoes [2, 3], including forest encephalitis virus, Rickettsia, Coxiella, Borrelia burgdorferi, Anaplasma, and Babesia [4, 5, $6,7,8]$. Depending on the pathogen the severity of tick-borne disease (TBD) can even be life-threatening. As human populations have grown and their interactions with the wild have increased, also human exposure to pathogens carrying ticks has greatly increased [9]. The transmission process of TBD is influenced by many factors, including pathogens, vectors, potential hosts, the environment, and human behavior. In addition, TBD often benefits from human population mobility, animal migration, and global logistics. Especially the global logistics increases the possibility of tick-borne pathogens spreading 
across borders [10]. Worldwide, tick-borne diseases (TBD) such as tick-borne encephalitis, CrimeanCongolese hemorrhagic fever, and Rocky Mountain spot fever have posed new threats to public health around the world, and the incidence of TBD is increasing at an alarming rate [11-13]. The United States reported nearly 650,000 cases of vector-borne diseases from 2004 to 2016 . More than $75 \%$ of these cases were tick-borne diseases. From 2012 to 2017 the United States reported nearly 288,000 cases of seven tick-borne diseases to the Centers for Disease Control and Prevention (CDC). Due to underreporting, the true number of cases may be higher [14]. At the same time, the rate of introduction or late identification of new or unknown tick-borne pathogens is also accelerating [15].

China has a vast territory, complex geography, diverse climate, and a wide variety of ticks. So tick-borne diseases are prevalent in most areas of China, seriously affecting human health [16, 17]. Zhao et al. [18] found that $D$. nuttalli is one of the tick species carrying many different tick-borne pathogens in China. According to the model prediction [18], the habitat suitable for 19 tick species was $14-476 \%$ larger than the geographical area where these species are currently found, indicating that there are still serious deficiencies in our knowledge of tick distribution. Due to large pasture areas and extensive animal husbandry, Inner Mongolia provides excellent habitat for ticks and the zoonosis incidence is often very high [19]. In 2005 Jia N et al. [20] reported human cases of Rickettsia raoultii in northeast China.

Ticks and tick-borne diseases have a substantial impact on the economy and human life in Inner Mongolia. Nevertheless, the community structure and diversity of microbial communities on ticks parasitizing sheep in various regions of Inner Mongolia have not been thoroughly studied so far. The microbial communities of ticks are influenced by many factors, including geographical area, feeding status, blood meal source, and developmental stage [21].

Current techniques for $16 \mathrm{~S}$ ribosomal RNA (rRNA) gene sequence analysis, based on typical clustering thresholds of operational taxa (OTUs), are insufficient for accurate taxonomic allocation and for addressing the phylogenetic relationships at the species level when only a few hypervariable regions are amplified. Therefore, 16S rRNA gene amplification sequence data using the v3-v4 region can only be explored at the genus level [22]. PacBio full-length 16S rDNA third-generation sequencing technology, however, is more accurate and can be used for precise species identification [23].

Therefore, we collected ticks from the surface of sheep in four main pastoral areas of Inner Mongolia, and analyzed their microbial diversity using PacBio full-length 16S rDNA third-generation sequencing technology. The aim of our investigation was to assess the distribution characteristics of tick microbial communities in different geographical locations in Inner Mongolia, providing information for better environmental management.

\section{Methods}

\section{Tick collection and Sample Preparation}


In mid-April 2019 a total of 941 ticks were collected from sheep in Hulun Buir City (New Barag Right Banner), Chifeng City (Bayan Wendusumu Area, Tianshan Town, Alukeerqin Banner), Ordos City (Chengchuan Town, Otog Front Banner), and the Forest area of Arshan. According to morphological identification, 905 D. nuttalli and 36 I. persuleatus (Table 1, Fig. 1, Fig. 2) were collected and stored at $-80^{\circ} \mathrm{C}$.

Table 1

Tick samples collection information.

\begin{tabular}{|lllll|}
\hline Location & Tick species & Longitude & Latitude & Samples \\
\hline Hulun Buir & D. nuttalli & $116^{\circ} 82^{\prime}$ & $48^{\circ} 67^{\prime}$ & 292 \\
\hline Chifeng & D. nuttalli & $121^{\circ} 64^{\prime}$ & $43^{\circ} 46^{\prime}$ & 543 \\
\hline Ordos & D. nuttalli & $108^{\circ} 32^{\prime}$ & $37^{\circ} 70^{\prime}$ & 70 \\
\hline Arshan & I. persuleatus & $119^{\circ} 94^{\prime}$ & $47^{\circ} 17^{\prime}$ & 36 \\
\hline
\end{tabular}

\section{DNA extraction}

The morphologically identified tick samples were disinfected with $75 \%$ ethanol, dried on filter paper, washed three times with PBS, and finally dried on filter paper.A total of 941 tick samples were divided into 206 sample pools. In each sample pool, the same area with the genus $5 \sim 20$ not only full blood or $1 \sim 2$ only full blood tick were taken and put into a sterile grinding tube, add $5 \mathrm{~mm}$ magnetic bead 1 and $3 \mathrm{~mm}$ magnetic beads 2 , grinding tube into the adapter (ahead $-20{ }^{\circ} \mathrm{C}$ refrigerator $30 \mathrm{~min}$ ), install the adapter into the grinding apparatus, set the parameter to $70 \mathrm{~Hz}$ and $180 \mathrm{~S}$. Finally, according to TIANGEN blood / cell / tissue genomic DNA extraction kit instructions to extract DNA, all extracted DNA stored at- $20^{\circ} \mathrm{C}$ standby. Three DNA samples ( Table 2 ) were randomly selected from each area, and $10 \mu \mathrm{L}$ of each sample was prepared for microbial diversity sequencing.

\section{S rRNA Amplicon Sequencing and Data Analysis}

The V1-V9 region of the bacteria $16 \mathrm{~S}$ ribosomal RNA gene were amplified by PCR $\left(95^{\circ} \mathrm{C}\right.$ for $2 \mathrm{~min}$, followed by 27 cycles at $95^{\circ} \mathrm{C}$ for $30 \mathrm{~s}, 55^{\circ} \mathrm{C}$ for $30 \mathrm{~s}$, and $72^{\circ} \mathrm{C}$ for $60 \mathrm{~s}$ and a final extension at $72^{\circ} \mathrm{C}$ for 5 min) using primers 27F 5'-AGRGTTYGATYMTGGCTCAG-3' and 1492R 5'-RGYTACCTTGTTACGACTT-3', where barcode is an eight-base sequence unique to each sample. PCR reactions were performed in triplicate $20 \mu \mathrm{L}$ mixture containing $4 \mu \mathrm{L}$ of $5 \times$ FastPfu Buffer, $2 \mu \mathrm{L}$ of $2.5 \mathrm{mM}$ dNTPs, $0.8 \mu \mathrm{L}$ of each primer $(5 \mu \mathrm{M}), 0.4 \mu \mathrm{L}$ of FastPfu Polymerase, and $10 \mathrm{ng}$ of template DNA. Amplicons were extracted from $2 \%$ agarose gels and purified using the AxyPrep DNA Gel Extraction Kit (Axygen Biosciences, Union City, CA, U.S.) according to the manufacturer's instructions.SMRTbell libraries were prepared from the amplified DNA by blunt-ligation according to the manufacturer's instructions (Pacific Biosciences). Purified SMRTbell libraries from the Zymo and HMP mock communities were sequenced on dedicated 
PacBio Sequel cells using the S/P1-C1.2 sequencing chemistry. Purified SMRTbell libraries from the pooled and barcoded samples were sequenced on a single PacBio Sequel cell. Replicate 1 of the samples was sequenced using the S/P2-C2/5.0 sequencing chemistry, and Replicate 2 of the samples was sequenced with a pre-release version of the S/P3-C3/5.0 sequencing chemistry. All amplicon sequencing was performed by Shanghai Biozeron Biotechnology Co. Ltd (Shanghai, China).

PacBio raw reads were processed using the SMRT Link Analysis software version 6.0 to obtain demultiplexed circular consensus sequence (CCS) reads with the following settings: minimum number of passes $=3$, minimum predicted accuracy $=0.99$. Raw reads were processed through SMRT Portal to filter sequences for length (<800 or $>2500 \mathrm{bp}$ ) and quality. Sequences were further filtered by removing barcode, primer sequences, chirmas and sequences if they contained 10 consecutive identical bases. OTUs were clustered with $97 \%$ similarity cutoff using UPARSE(version $7.1 \mathrm{http}: / /$ drive5.com/uparse/) and chimeric sequences were identified and removed using UCHIME. The phylogenetic affiliation of each $16 S$ rRNA gene sequence was analyzed by RDP Classifier (http://rdp.cme.msu.edu/) against the silva (SSU132)16S rRNA database using confidence threshold of 70\% [24].Subsequently Alpha and Betadiversity Analyses.The rarefaction analysisbased on Mothur v.1.21.1[25] was conducted to reveal the diversity indices, including the Chao, ACE, and Shannon diversity indices. The beta diversity analysis was performed using UniFrac [26] to compare the results of the principal component analysis (PCA) using the community ecology package, R-forge (Vegan 2.0 package was used to generate a PCA figure).One way analysis of variance (ANOVA) tests were performed to assess the statistically significant difference of diversity indices between samples. Differences were considered significant at $p<0.05$. Venn diagrams were drawn using online tool “Draw Venn Diagram” (http://bioinformatics.psb.ugent.be/webtools/Venn) to analyze overlapped and unique OTUs during the treatment processes.

For identification of biomarkers for highly dimensional colonic bacteria, LEfSe (linear discriminant analysis effect size) analysis was done [27]. Kruskal-Wallis sum-rank test was performed to examine the changes and dissimilarities among classes followed by LDA analysis to determine the size effect of each distinctively abundant taxa [28].

\section{Results}

\section{PacBio sequencing data}

A total of 12 samples were sequenced (Table 2). After data screening and deletion, a total of 20,1159 reads were generated and classified. The reads of each sample ranged from 11,550 to 21996 . The dilution curve of the Shannon index at OTU level showed a suitable range for sequencing, and the observed Shannon index accumulation curve also reached a plateau (Fig.3).

Table 2 Information of tick samples used for bacterial microbiome analysis. 


\begin{tabular}{|lll|}
\hline Sample Name & Tick Species & $\mathrm{n}$ \\
\hline Hulun Buir-1 & 8 \\
Hulun Buir-2 & 1 \\
Hulun Buir-3 & 7 \\
Chifeng-1 & 8 \\
Chifeng-2 & D. nuttalli & 10 \\
Chifeng-3 & 1 \\
Ordos-1 & 1 \\
Ordos-2 & 1 \\
Ordos-3 & 1 \\
1.persuleatus-1 & 6 \\
2. persuleatus-2 & I. persuleatus & 6 \\
I.persuleatus-3 & & 6 \\
\hline
\end{tabular}

\section{Bacterial microbiome composition}

A total of 326 OTUs were detected in 12 samples, and the bacterial microbial components were 11 phyla, 15 classes, 38 orders, 62 families, 104 genera, and 141 species. At the genus level, the abundance of Rickettsia was the highest (35.27\%), followed by Ac37b (19.33\%), Arsenophonus (11.21\%), Candidatus Lariskella (10.84 \%), Acinetobacter (7.17\%), Cupriavidus (2.39\%), and Romboutsia (1.00\%). Most Ac37b was found in the $D$. nuttalli of Ordos and Chifeng, while Candidatus Lariskella mainly existed in the I. persulcatus. At the species level, Rickettsia raoultii had the highest abundance (23.23\%), followed by Ac37b unclassified (19.33\%). Rickettsia unclassified (11.94\%), Candidatus Lariskella unclassified (10.84\%), Arsenophonus unclassified (10.32\%). Rickettsia raoultii exists in most samples but is rarely found in Ordos.

\section{Differences of ticks in different areas}

There were 180, 182 and 135 OTUs in the samples of D. nuttalli from Hulun Buir, Chifeng and Ordos, respectively. According to the a diversity the Hulun Buir region shows a greater microbial diversity than the other two regions (fig. 5). At the genus level, there were Rickettsia (35.87\%), Ac37b (0.05\%) and Arsenophonus (41.51\%) in the Hulun Buir region. In Chifeng area, Rickettsia (57.42\%), Ac37b (16.38\%) and Arsenophonus (3.32\%) were found. The Ordos region had Rickettsia (0.08\%), Ac37b (60.74\%) and Arsenophonus (0.01\%). To further distinguish the composition of the microbial community, weighted UniFrac analyses revealed differences regarding the region, as measured by an analysis of similarity (ANOSIM, R=0.7994, $\mathrm{P}=0.011$ ) and visualized by principal component analysis (PCoA) (Fig. 6). PCoA 
explained $47.46 \%$ (Axis 1) and $28.93 \%$ (Axis 2) of the variation, with samples from different regions clustering separately (Fig. 6).

\section{Differences Between D. nuttalli and I. persuleatus}

North of the Arshan region is adjacent to the New Barag Left Banner and to the Ewenki Autonomous Banner of the Hulun Buir City, close to the Hulun Buir tick collection point. All I. persuleatus came from the Arshan region. Whereas in the Hulun Buir area only D. nuttalli was found. A total of 123 microbial OTUs were found in ticks, and a diversity indicated that specimens of $D$. nuttalli contained greater microbial diversity than specimens of $I$. persuleatus (Fig. 5). Among the first 10 genera, there were significant differences between the microbial compositions of I. persuleatus and D. nuttalli. Rickettsia (47.71\%), Candidatus Lariskella (43.34\%), and Acinetobacter (1.71\%) were the major microbial components of I. persuleatus. Rickettsia (35.87\%), Arsenophonus (41.51\%) and Cupriavidus (5.66\%) were found in D. nuttalli.

Among the first 10 species, the Rickettsia unclassified (45.37\%), Rickettsia raoultii (1.96\%) and Candidatus Lariskella unclassified (43.34\%) were the main microorganisms in I. persuleatus, while Rickettsia raoultii (34.99\%), Arsenophonus unclassified (38.12\%) and Cupriavidus gilardii CR3 (5.66 \%) were the main microorganisms in $D$. nuttalli. The differences in the microbial composition were significant.

In general, D. nuttalli in Hulun Buir area showed the highest microbial diversity. We selected pathogenic bacteria with high abundance and obvious harm to humans at the species level (Rickettsia raoultii, Anaplasma, Rickettsia bellii, Coxiella) for further analysis. We found that Ac37b, a new type of Rickettsia, was predominant in the Ordos region, and Anaplasma was much more abundant in the Ordos region than in other regions. The abundance of Rickettsia was very low in Ordos, but it was higher in D. nuttallifrom Hulun Buir and Chifeng. Rickettsia bellii was detected for the first time in Inner Mongolia. It only appeared in the I. persuleatus group and there were many unclassified Rickettsia. Arsenophonus unclassified was also an important component of its microorganisms in Hulun Buir (Fig.7).

\section{Discussion}

D. nuttalli is a tick species widely distributed in Inner Mongolia, eastern Siberia, and China. It is parasitic on livestock and can also cause damage to humans [29]. D.nuttalli can carry different pathogens including Babesia, Anaplasma vois, Rickettsia, and Coxellia [30, 31, 32]. I. persuleatus is the dominant tick species in northeast China. Inner Mongolia has a large east-west span, so there are I. persuleatus populations at the border with northeast China. This often is associated with the transmission of tickborne encephalitis virus and can pose a serious threat to human life and safety [33,34].

D. nuttalli is the dominant tick species in Inner Mongolia, with significant influence on the economy and health of the local human population. Jiao et al. [35] carried out a simple microbial diversity analysis of ticks on cattle in the Hulun Buir area of Inner Mongolia. Beyond this, the microbial community 
composition of ticks in other areas of Inner Mongolia was not further investigated. The bacterial diversity on different tick species must be analyzed to further understand the relationships between ticks and microorganisms. Samples collected from multiple regions are more likely to find new pathogens in microbial diversity.

In our investigation, we applied PacBio full-length 16S rRNA third-generation sequencing to the V1-V9 region of the 16S rRNA. In their study on oral microorganisms, Zhang et al. [36] found that OTU sequences generated by PacBio were much larger than those generated on the MiSeq platform. Therefore, we adopted PacBio full-length 16S rRNA third-generation sequencing for the microbial diversity analysis of ticks in Inner Mongolia. According to our analysis, the microbial diversity on $D$. nuttalli samples from different regions of Inner Mongolia was significantly different. The highest microbial diversity was found in D. nuttalli samples from Hulun Buir, and Rickettsia (35.87\%) and Arsenophonus (41.51\%) were the main microorganisms. Rickettsia is an arthropod-associated obligate intracellular gram-negative bacterium that can cause mild to severe disease in humans [37]. Arsenophonus is an intracellular symbiotic bacterium of insects with a wide host range and rich biodiversity. An androidal effect on ticks has not been reported so far, and its other biological functions have not yet been identified [38].

Linear discriminant analysis effect size (LEfSe) analysis showed that Rickettsia raoultii, Peptostreptococcaceae and Clostridia played an important role in the Chifeng formation. Characteristic of the Ordos formation is Anaplasma. Enterobacterale and Xanthomonadaceae belong to Hulun Buir (Fig. 8). Rickettsia raoultii is the pathogene of the spotted fever group, which is transmitted vertically in arthropods as a symbiotic bacterium and in vertebrates as a pathogenic bacterium and is a pathogen of human diseases [39]. Anaplasma is a gram-negative intracellular obligate parasite, and its pathogenicity poses an important threat to several animals, and even public health security [40]. Currently, there are six species of Anaplasma recognized worldwide, including $A$. phagocytophilum, A. ovis, A. capra, A. bovis, A. marginale and $A$. platys [41]. In addition to $A$. phagocytophilum, $A$. bovis and $A$. capra have been reported to infect humans $[42,43]$. A novel tyhpus group Rickettsia species ( $R$. bacterium Ac37b) was found in the Ordos region [44]. The Rickettsia typhus group is mainly composed of $R$. przewskii and $R$. morzewskii. In Australia, three types of typhus, epidemic typhus, mouse typhus, and tsutsugamushi disease, have been found successively. These are closely related to native wild animals and ticks in Australia [45]. In China, typhus has also been an important cause of human morbidity and mortality in the past decade. The disease was initially identified only in southern China, but now cases of typhus have been reported in northern China, with a wide geographical distribution [46].

$R$. bellii is the only known species in a third group that differentiated before the spotted fever group and the typhus group separated. $R$. bellii is the most common Rickettsia in American ticks and is found in all species of Ixodes. Including Dermacentor and Amblyomma, it is also the only Rickettsia that has been found in both Ixodes, showing the largest arthropod host range among known Rickettsia: It is pathogenic to mammals [47]. But the pathogenic potential for humans is still unknown and should be closely monitored [48]. 
We can further explore the relationship between Rickettsia bellii and other Rickettsia. In the Ordos area, there were pathogenic bacteria that are more threatening to humans, and a new classification of Rickettsia has emerged. The pathogen prevention and control in this area needs to focus on monitoring and strengthening the popular knowledge of methods for personal protection. The newly discovered pathogens in Inner Mongolia need to be isolated and sequenced in future studies, and the pathogenicity of the organisms should be tested through subsequent animal experiments. At the same time, the sampling sites can be expanded in future investigations, and ticks should be collected from different hosts, such as cattle, camels, and other mammals.

\section{Conclusions}

In this study, we analyzed the microbial diversity of two ticks in four regions of Inner Mongolia. A novel rickettsia species (R.bacterium Ac37b ) was found in Inner Mongolia for the first time, and Rickettsia Bellii was found in I.persuleatus. Ticks carried more potential pathogens in Ordos area,and there were coinfections of Rickettsia and Anaplasma, which may be related to its geographical environment. The prevention and control of tick-borne diseases in this region should be strengthened. In the future research, sampling in the western region of Inner Mongolia can be focused on.

\section{Abbreviations}

D. nuttalli:Dermacentor nuttalli,l.persuleatus:Ixodes persuleatus;PCR:

Polymerase chain reaction;R.bacterium Ac37b:Rickettsia bacterium Ac37b

\section{Declarations}

\section{Acknowledgements}

We are very grateful to the Inner Mongolia Center for Disease Control and Prevention for providing tick samples. We are very grateful to the Molecular Biology Research Center of Inner Mongolia Medical University for providing experimental facilities and conditions.

\section{Authors' contributions}

LW,LLX ,ZG and SS performed laboratory analysis, analyzed the data and wrote the frst draft. JFY,DDQ and LM revised the manuscript. XYS participated in sample collection. SYF directed the experiment and helped to revise the manuscript. All authors read and approved the final manuscript.

\section{Funding}

This work received financial support from the "Achievement transformation" project of Inner Mongolia Medical University(Grant YKD2020CGZH001),Zhiyuan talent project of Inner Mongolia Medical University(Grant ZY0201027). 
Availability of data and materials

All datasets have been included with this article.

Ethics approval and consent to participate.

Not applicable.

\section{Consent for publication}

Not applicable.

\section{Competing interests}

The authors declare that they have no competing interests.

\section{Author details}

${ }^{1}$ Graduate School, Inner Mongolia Medical University, Hohhot 010059,

Inner Mongolia, China. ${ }^{2}$ Department of Parasitology, Inner Mongolia Medical University, Hohhot 010110 , Inner Mongolia, China. ${ }^{3}$ Hulunbuir Mental Health Center,Hulunbuir 022150,Inner Mongolia,China. ${ }^{4}$ Inner Mongolia Academy of Agricultural \& Animal Husbandry Science, Hohhot 010031, Inner Mongolia, China. ${ }^{5}$ Inner Mongolia Center for Disease Control and Prevention, Hohhot 010000, Inner Mongolia, China. ${ }^{6}$ Ningbo Institute of Life and Health Industry, University of Chinese Academy of Sciences, Ningbo 315010, China. ${ }^{7}$ Key Laboratory of Diagnosis and Treatment of Digestive System Tumors of Zhejiang Province, Hwa Mei Hospital, University of Chinese Academy of Sciences, Ningbo 315010, China.

\section{References}

[1] Mead P, Hinckley A, Hook S, Beard CB. TickNET-A Collaborative Public Health Approach to Tickborne Disease Surveillance and Research. Emerging Infectious Diseases, 2015; 21(9):1574-1577.

[2] Colwell D D , Dantas-Torres F , Otranto D . Vector-borne parasitic zoonoses: Emerging scenarios and new perspectives. Veterinary Parasitology, 2011; 182(1):14-21.

[3] F. JONGEJAN, G. UILENBERG. The global importance of ticks. Parasitology, 2004;129.

[4] Sasaki K, Honma, M, NakaoM , Sasaki M, Yoshii K. Survey to detect tick-borne encephalitis virus from human-feeding ticks in hokkaido, japan. The Journal of Dermatology. 2021.

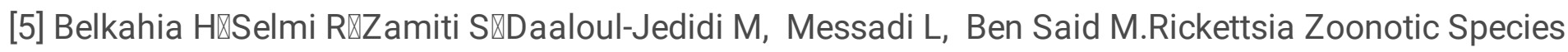
in Small Ruminant Ticks From Tunisia.Front Vet Sci .2021;8:676896. 
[6] Körner S, Makert GR, Ulbert S,Pfeffer M, Mertens-Scholz K.Coxiella burnetiiThe Prevalence of in Hard Ticks in Europe and Their Role in Q Fever Transmission Revisited-A Systematic Review.Front Vet Sci .2021;8:655715.

[7]Yuan-Zhi,Wang,Lu-Meng, Yang MH, Zhang L, Du JY, Liu ZQ, Li YX, Lu WH, Chen CF, Wang Y, Chen RG, Xu J, Yuan L, Zhang WJ, Zuo WZ, Shao RF. A broad-range survey of ticks from livestock in Northern Xinjiang: changes in tick distribution and the isolation of Borrelia burgdorferi sensu stricto. Parasites \& Vectors. 2015.

[8] Koseoglu A E , Hüseyin Can, Mervenur Güvendi, et al. Molecular investigation of bacterial and protozoal pathogens in ticks collected from different hosts in Turkey. Parasites \& Vectors.2021;14(1).

[9] Simon J A, Marrotte R R, Desrosiers N , Erkunt Alak S, Kandemir Ç, Taşkın T, Demir S, Akgül G, Değirmenci Döşkaya A, Karakavuk M, Döşkaya M, Gürüz AY, Ün C. Climate change and habitat fragmentation drive the occurrence of Borrelia burgdorferi, the agent of Lyme disease, at the northeastern limit of its distribution. Evolutionary Applications. 2015; 7(7):750-764.

[10] Kayoko Y , Hiroka A , Hirotaka K . Distribution of tick-borne diseases in Japan: Past patterns and implications for the future. Journal of Infection \& Chemotherapy. 2018;24(7):499-504.

[11] Randolph S E . Tick-borne encephalitis incidence in Central and Eastern Europe: consequences of political transition. Microbes \& Infection.2008; 10(3):209-216.

[12] Maltezou H C , Andonova L , Andraghetti R, Bouloy M, Ergonul O, Jongejan F, Kalvatchev N, Nichol S, Niedrig M, Platonov A, Thomson G, Leitmeyer K, Zeller H. Crimean-Congo hemorrhagic fever in Europe: current situation calls for preparedness.2010; 15(10):19504.

[13] DLF José, Waterhouse R M , Sonenshine D E, Roe RM, Ribeiro JM, Sattelle DB, Hill CA. Tick Genome Assembled: New Opportunities for Research on Tick-Host-Pathogen Interactions. Frontiers in Cellular and Infection Microbiology.2016; 6:103-.

[14] Ronald R, Lindsey NP, Fischer M,Gregory CJ, Hinckley AF, Mead PS, Paz-Bailey G, Waterman SH, Drexler NA, Kersh GJ, Hooks H, Partridge SK, Visser SN, Beard CB, Petersen LR. Vital signs: trends in reported vectorborne disease cases-United States and territories, 2004-2016. MMWR Morb Mortal Wkly Rep. 2018;67(17): 496-501.

[15] Vital Signs: Trends in Reported Vectorborne Disease Cases - United States and Territories, 20042016.

[16] Wang GL, Huang J, Gong XH.Aishajiang.Abula: Hard tick and tick-borne diseases. Xinjiang Xu Mu Ye.2010;9:48-50.

[17] Zhao JW, Wang H-Y, Wang Y.Regional distribution profiles of tick-borne pathogens in China. Chinese Journal Biology and Control. 2012;5:445-447. 
[18] Zhao G P , Wang Y X, Fan Z W , Ji Y , Fang L Q. Mapping ticks and tick-borne pathogens in China. Nature Communications.2021; 12(1):1075.

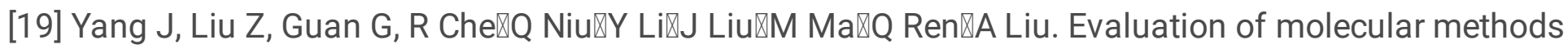
for detection of Borrelia burgdorferi senso lato in ticks. Diagn Microbiol Infect Dis.2012;73(1): 80-83.

[20]Jia N, Zheng YC, Ma L, Huo QB, Ni XB, Jiang BG, Chu YL, Jiang RR, Jiang JF, Cao WC. Human infections with Rickettsia raoultii, China. Emerg Infect Dis. 2014;20:866-8.

[21] Ruiling Z, Zhendong H, Guangfu Y, Zhong Z. Characterization of the bacterial community in Haemaphysalis longicornis (Acari: Ixodidae) throughout developmental stages. Exp Appl Acarol. 2019;77(2):173-186.

[22] Poirier S, Rué O, Peguilhan R, Coeuret G, Zagorec M, Champomier-Vergès MC, et al. Deciphering intraspecies bacterial diversity of meat and seafood spoilage microbiota using gyrB amplicon sequencing: a comparative analysis with 16S rDNA V3-V4 amplicon sequencing. PLoS One. 2018;13(9):e0204629.

[23] Esther Singer,Brian Bushnell,Devin Coleman-Derr,Brett Bowman,Robert M Bowers,Asaf Levy,Esther A Gies,Jan-Fang Cheng,Alex Copeland,Hans-Peter Klenk,Steven J Hallam,Philip Hugenholtz,Susannah G Tringe,Tanja Woyke. High-resolution phylogenetic microbial community profiling. The ISME Journal.2016;10(8).

[24] Katherine R Amato, Carl J Yeoman, Angela Kent, et al. Habitat degradation impacts black howler monkey (Alouatta pigra) gastrointestinal microbiomes. The ISME Journal 2013; 7:1344-1353.

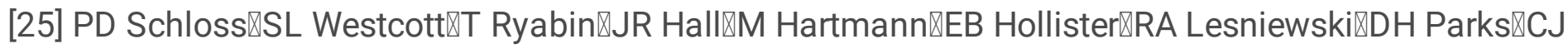
Robinson.Introducing mothur: open-source, platform-independent, community-supported software for describing and comparing microbial communities. Appl Environ Microbiol.2009;75:7537-7541.

[26] Lozupone C, Lladser ME, Knights D, Stombaugh J, Knight R.UniFrac: an effective distance metric for microbial community comparison. The ISME Journal.2011;5:169.

[27] Segata N, Izard J, Waldron L, Gevers D, Miropolsky L, Garrett W S. Metagenomic biomarker discovery and explanation. Genome Biol.2011;12:R60.

[28]ljaz MU, Ahmed MI, Zou X, Hussain M, Zhang M, Zhao F, Xu X, Zhou G, Li C.Beef, Casein, and Soy Proteins Differentially Affect Lipid Metabolism, Triglycerides Accumulation and Gut Microbiota of HighFat Diet-Fed C57BL/6J Mice. Frontiers in Microbiology. 2018;9.

[29] Kulakova N V , Khasnatinov M A, Sidorova E A , RV Adel Shin $\varangle S I$ Belikov. Molecular identification and phylogeny of Dermacentor nuttalli (Acari: Ixodidae).Parasitology Research.2014; 113(5):1787-1793. 
[30] Song R , Wang Q , Guo F , X Liu囚Y Wang. Detection of Babesia spp. Theileria spp. and Anaplasma ovis in Border Regions, northwestern China. Transboundary \& Emerging Diseases. 2018;65(1).

[31]Yin X, Guo S, Ding C, Cao M, Kawabata H, Sato K, Ando S, Fujita H, Kawamori F, Su H, Shimada M, Shimamura Y, Masuda S, Ohashi N,Spotted Fever Group Rickettsiae in Inner Mongolia, China, 20152016.Emerg Infect Dis. 2018;11;24(11).

[32]Ni J, Lin H, Xu X, Ren Q, Aizezi M, Luo J, Luo Y, Ma Z, Chen Z, Tan Y, Guo J, Liu W, Qu Z, Wu Z, Wang J, Li Y, Guan G, Luo J, Yin H, Liu G.

Coxiella burnetii is widespread in ticks (Ixodidae) in the Xinjiang areas of China.BMC Vet Res. 2020;28;16(1).

[33] Ma B, Ma XY, Zhang Y, Chen HB, Wang Q, Li LH.Ixodes persulcatus [Prediction of suitable habitats of in China].Zhongguo Xue Xi Chong Bing Fang Zhi Za Zhi_2021;30;33(2).

[34] M Liková, SF Havlíková, M Sláviková, M Slovák囚B Klempa. Dermacentor reticulatus is a vector of tickborne encephalitis virus.Ticks and Tick-borne Diseases.2020;11(4):101414.

[35] Jiao J , Lu Z, Yu Y, Yangxuan Ou凶Xiaolu Xiong. Identification of tick-borne pathogens by metagenomic next-generation sequencing in Dermacentor nuttalli and Ixodes persulcatus in Inner Mongolia, China. Parasites \& Vectors.2021; 14(1).

[36] Zhang J, Su L, Wang Y, Deng S.Improved High-Throughput Sequencing of the Human Oral Microbiome: From Illumina to PacBio.Can J Infect Dis Med Microbiol.2020.

[37] Raoult D, Roux V.Rickettsioses as paradigms of new or emerging infectious diseases.Clin Microbiol Rev.1997;10(4).

[38] CHENYU $\triangle W A N G W e i-x i a \triangle C H E N Y a n g \triangle F U Q i a n g$. Research progress on the bacterial symbiont Arsenophonus of insects.Zhejiang Nong Ye Xue Bao.2014; 26(2):0-530.

[39] Zhang J , Zhang Y B , Xue Y T ,H Liu囚GF Zhang®FH Wan. Research advances on a secondary endosymbiont Rickettsia in insect. Journal of Environmental Entomology.2017.

[40] Said M B , Belkahia H, Messadi L. Anaplasma spp. in North Africa: A review on molecular epidemiology, associated risk factors and genetic characteristics. Ticks and Tick-borne Diseases.2018;543-555.

[41] Kocan K M,de la Fuente J,Cabezas-Cruz A. The genus Anaplasma: new challenges after reclassification. Revue scientifique et technique (International Office of Epizootics).2015;34(2).

[42]Chochlakis D , loannou I, Tselentis Y , A Psaroulaki. Human Anaplasmosis and Anaplasma ovis Variant. Emerging Infectious Diseases.2010;16(6):1031-1032. 
[43] Li H , Zheng Y C , Ma L , et al. Human infection with a novel tick-borne Anaplasma species in China: a surveillance study. The Lancet Infectious Diseases.2015;15(6):663-670.

[44] Shpynov S N, Fournier P E, Pozdnichenko N N , AS Gumenuk $\ A A$ Skiba. New approaches in the systematics of rickettsiae. New Microbes and New Infections.2018;23:93-102.

[45] Graves, S.; Stenos, J. Rickettsioses in Australia. Ann. N. Y. Acad. Sci. 2009;1166:151-155.

[46] Lu M , Li F , Liao Y , JJ Shen $₫ Y Z$ Zhang. Epidemiology and Diversity of Rickettsiales Bacteria in Humans and Animals in Jiangsu and Jiangxi provinces, China. Scientific Reports. 2019; 9(1):1-9.

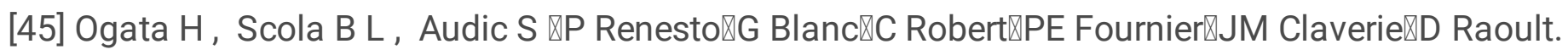
Genome Sequence of Rickettsia bellii Illuminates the Role of Amoebae in Gene Exchanges between Intracellular Pathogens. PLOS Genetics.2006;2(5):e76-.

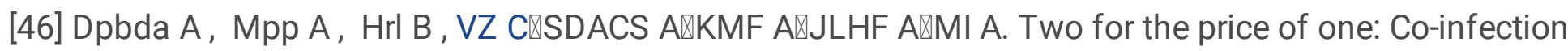
with Rickettsia bellii and spotted fever group Rickettsia in Amblyomma (Acari: Ixodidae) ticks recovered from wild birds in Brazil. Ticks and Tick-borne Diseases.2019;10( 6):101266-101266.

\section{Figures}




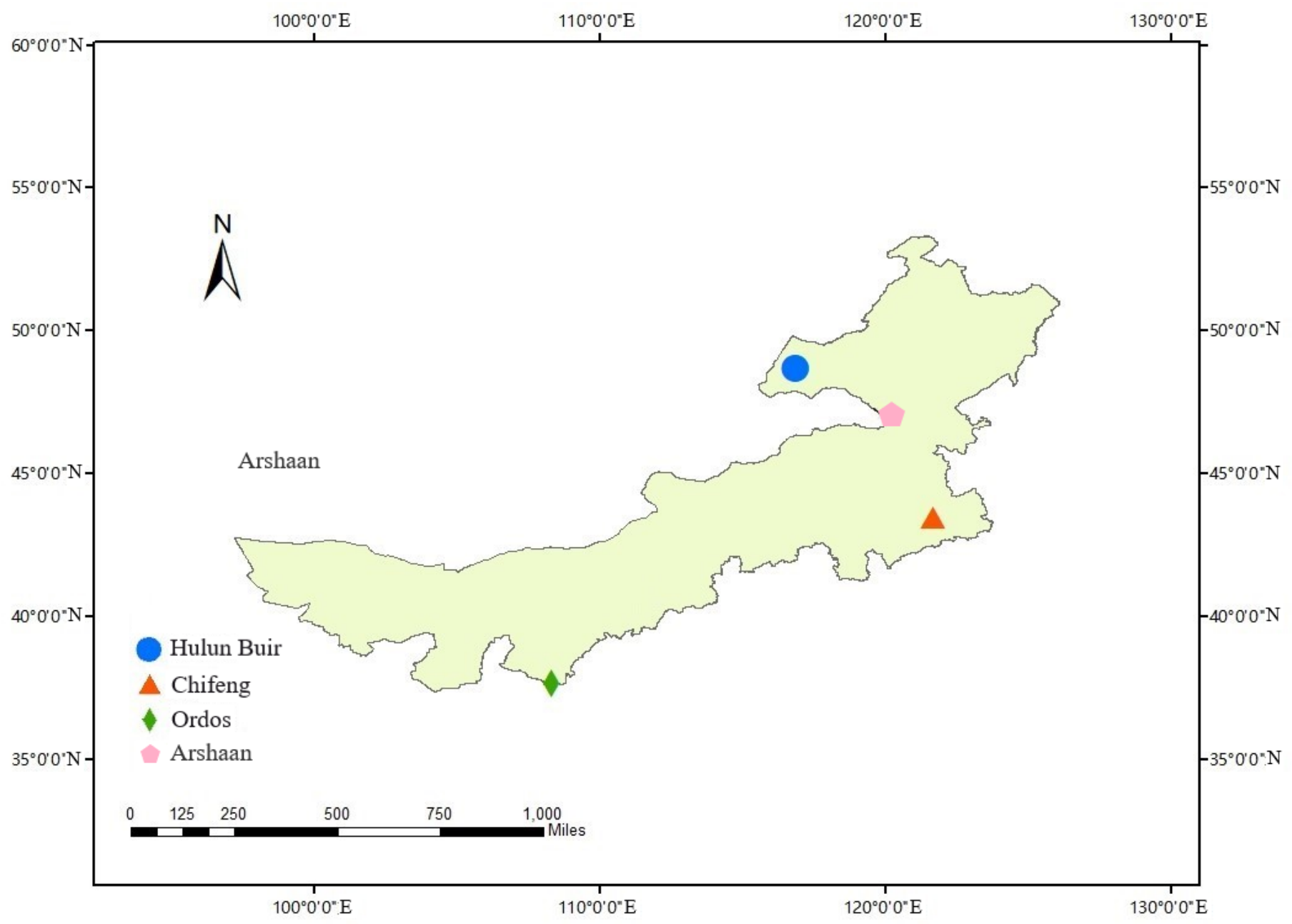

Figure 1

Map of tick collection sites in Inner Mongolia, China.

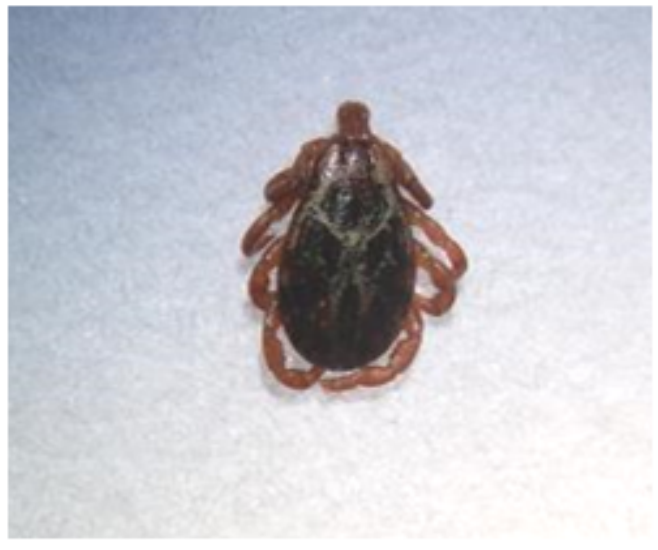

a

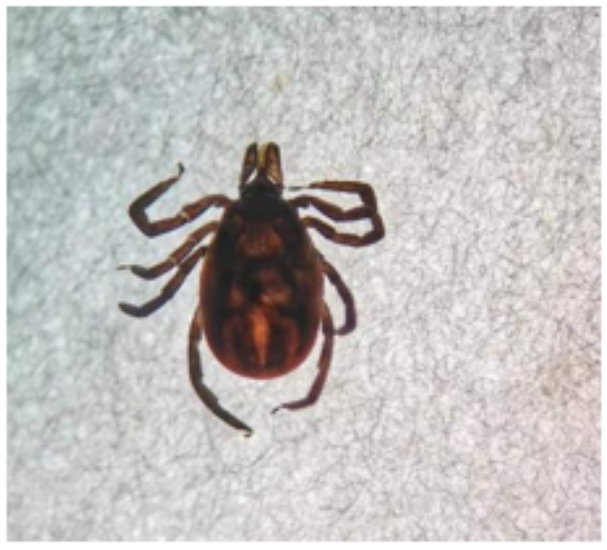

b

\section{Figure 2}

a Not full of blood D. nuttalli. b Not full of blood I. persuleatus. 


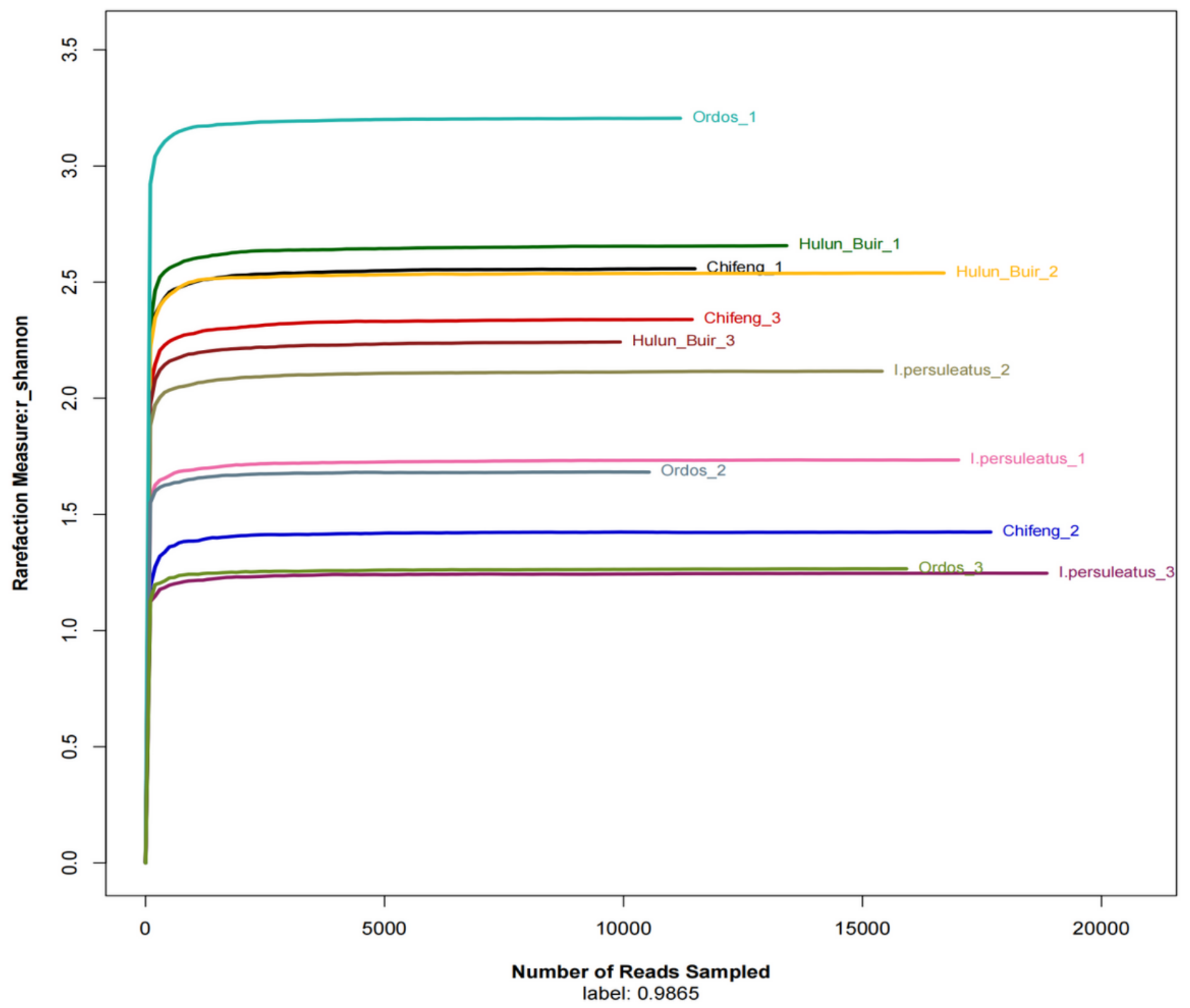

Figure 3

Shannon Wiener curve. 
(a)

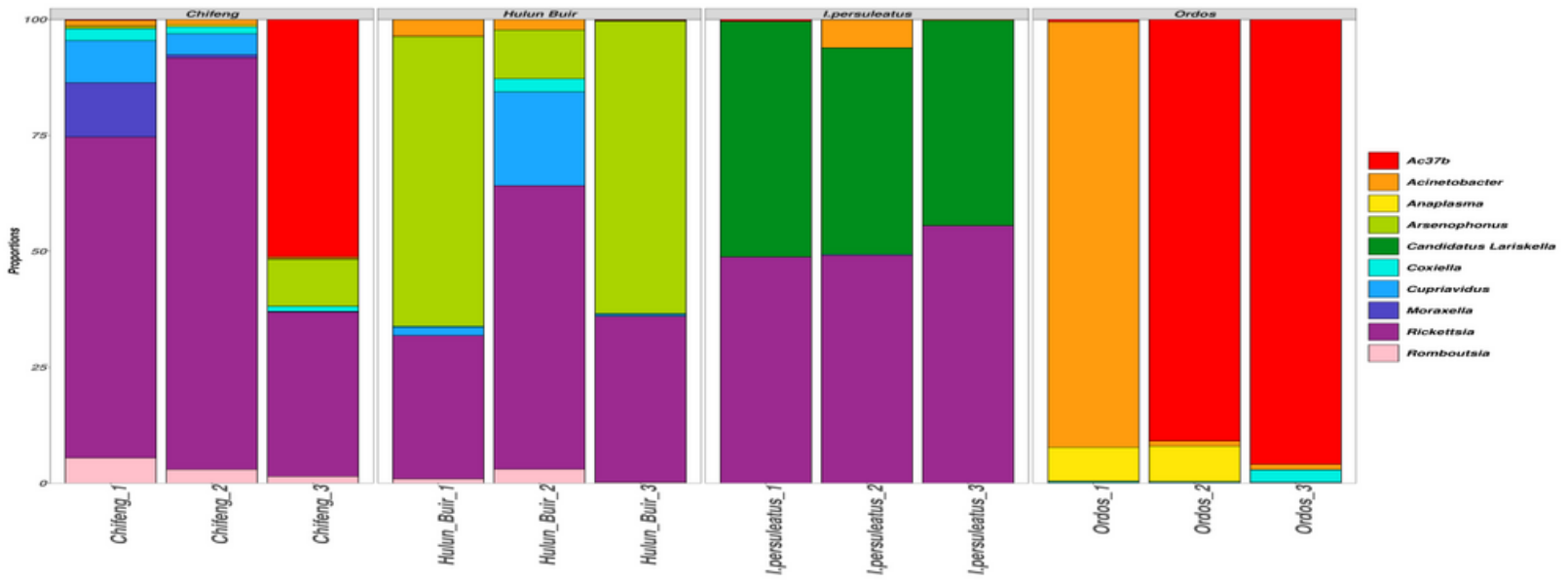

(b)

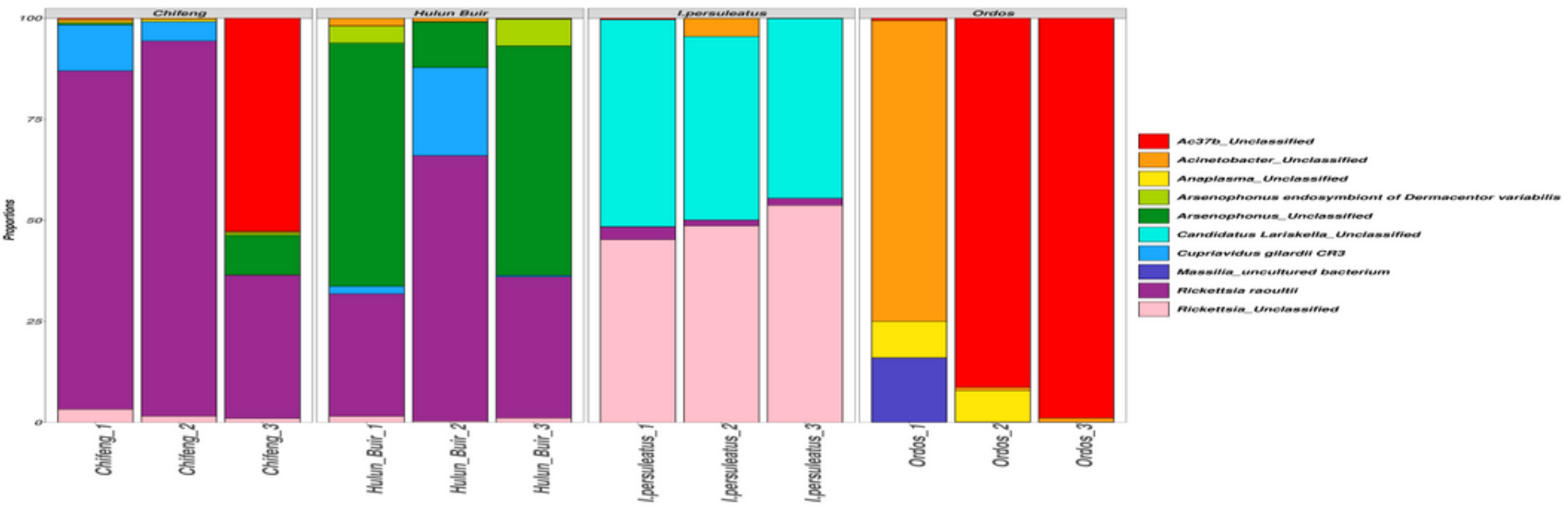

Figure 4

Microbe composition of different regions and species. a Top 10 microbial components at genus level. b Top 10 microbial components at species level. 
a

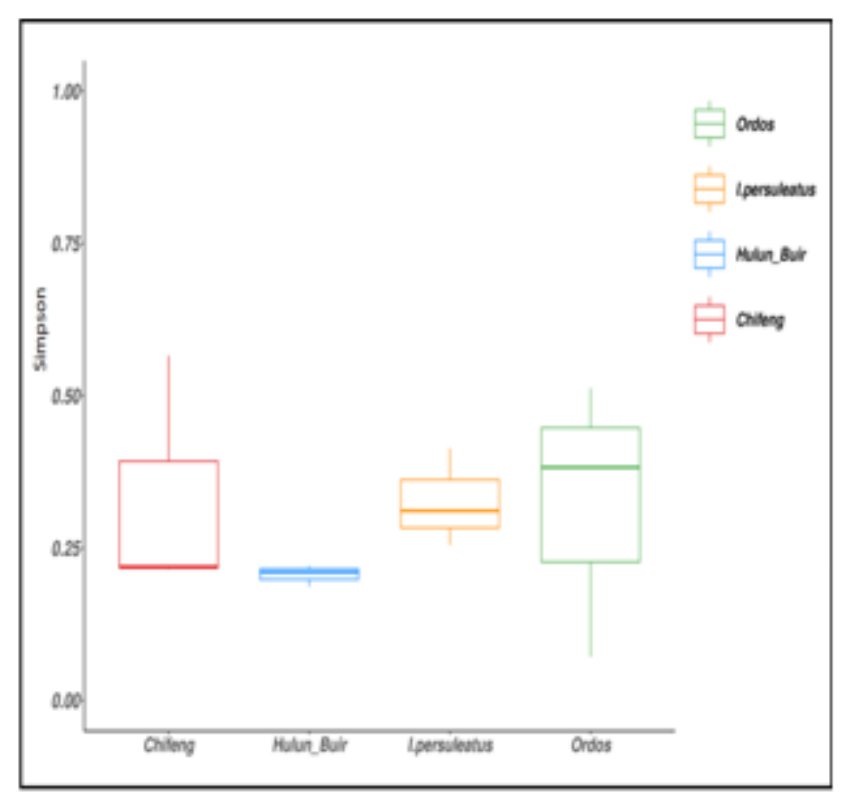

b

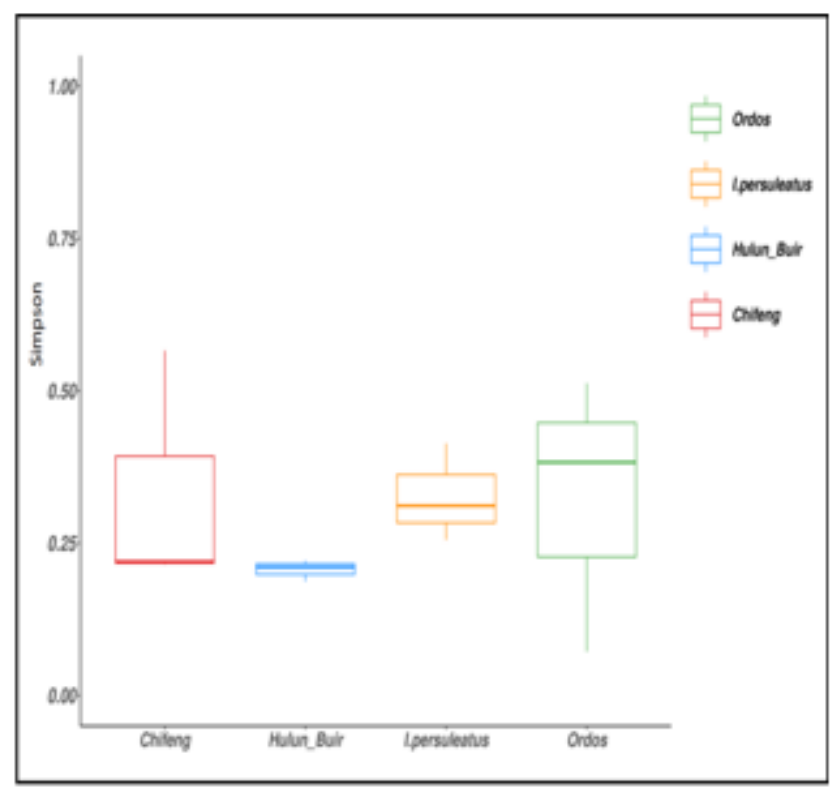

Figure 5

Alpha diversity measures for D. nuttalli and I. persuleatus in three areas. a Shannon's index. b Simpson's index 


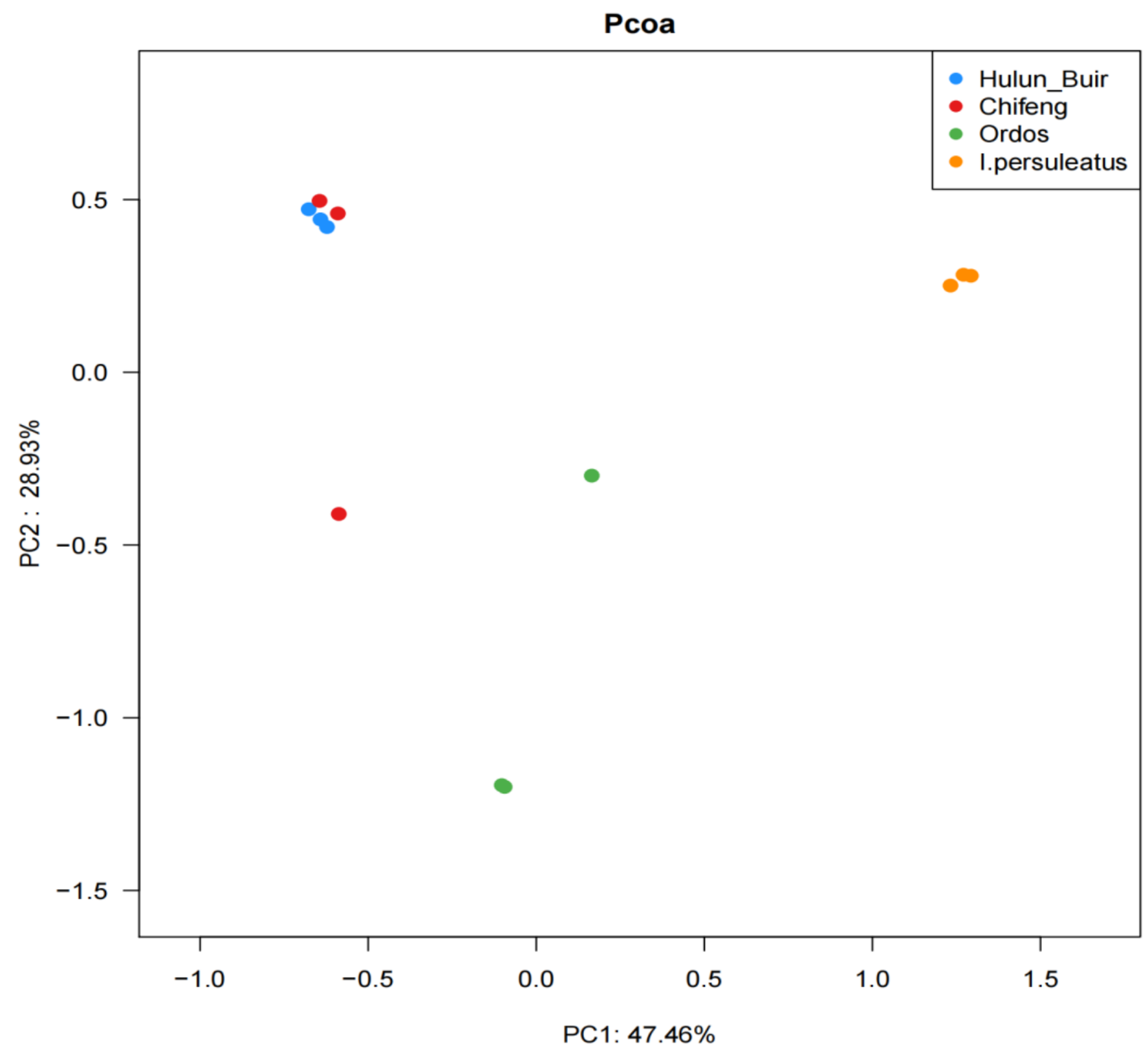

Figure 6

PCoA of $\beta$-diversity measures for twelve groups. Weighted UniFrac PCoA graph showing PC1, which accounts for $47.46 \%$ variation and PC2 for $28.93 \%$ variation. Different color dots represent different regions and species. 


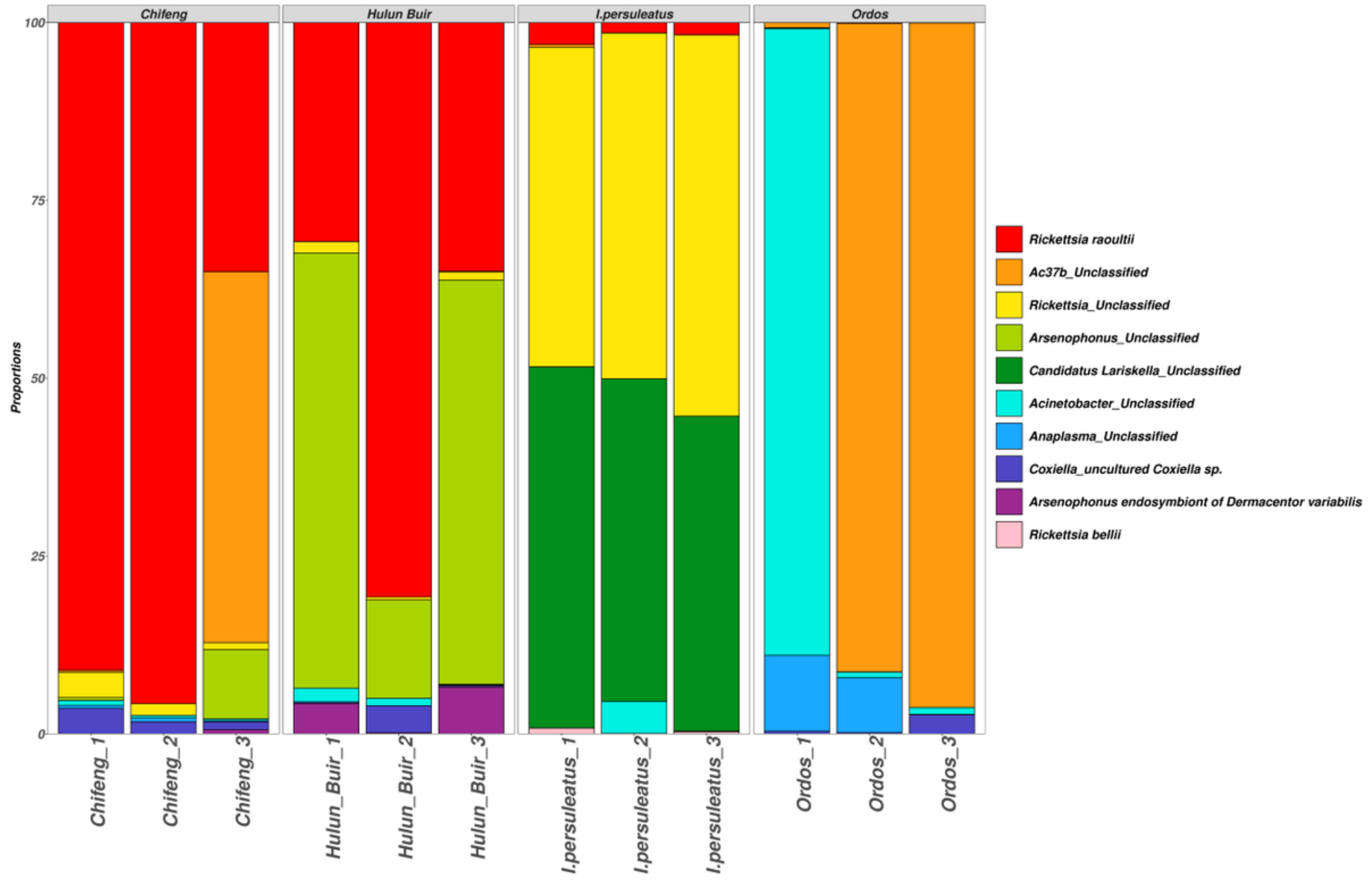

Figure 7

The composition of bacteria and pathogenic bacteria with a high abundance. 


\section{Cladogram}

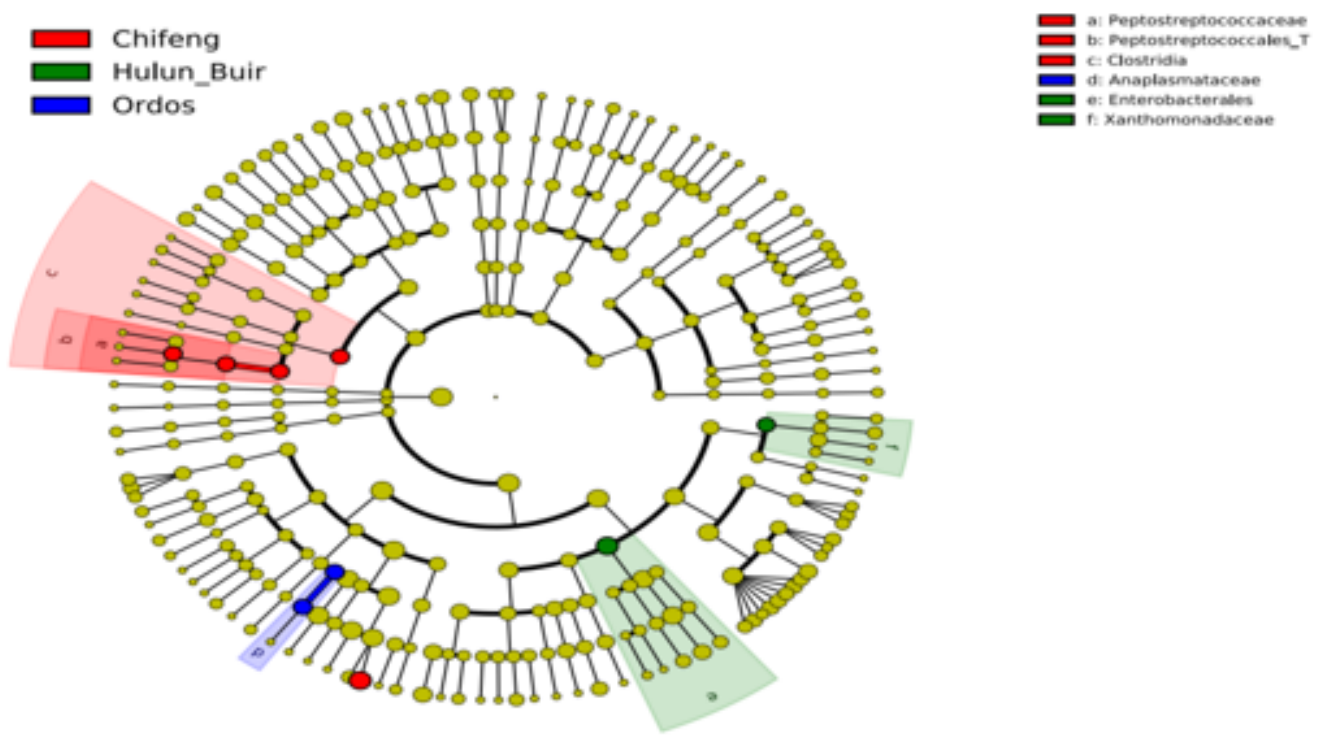

b

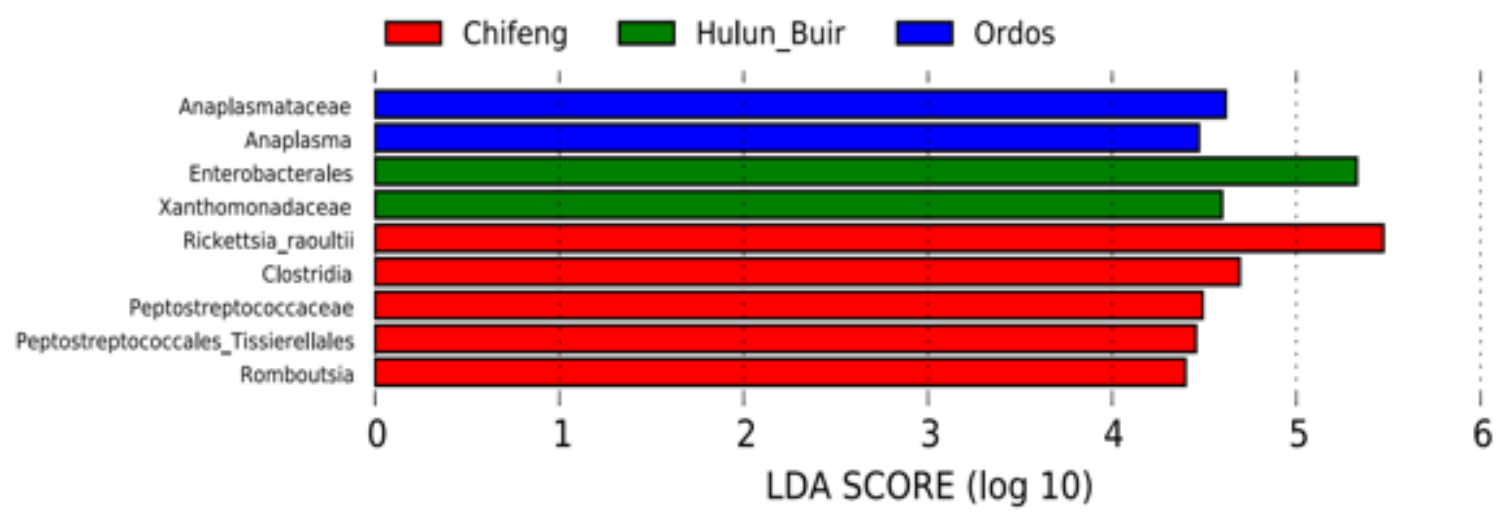

Figure 8

a Clustering tree analysis by Linear discriminant analysis effect size (LEfSe). b Histogram of LDA analysis

\section{Supplementary Files}


This is a list of supplementary files associated with this preprint. Click to download.

- renamed7c5c4.jpg 